\title{
A Comparative Study on the Ability of Two Implicit Solvent Lipid Models to Predict Transmembrane Helix Tilt Angles
}

\author{
Aaron Frank · Ioan Andricioaei
}

Received: 18 September 2010/Accepted: 5 November 2010/Published online: 9 December 2010

(C) The Author(s) 2010. This article is published with open access at Springerlink.com

\begin{abstract}
Free-energy profiles describing the relative orientation of membrane proteins along predefined coordinates can be efficiently calculated by means of umbrella simulations. Such simulations generate reliable orientational distributions but are difficult to converge because of the very long equilibration times of the solvent and the lipid bilayer in explicit representation. Two implicit lipid membrane models are here applied in combination with the umbrella sampling strategy to the simulation of the transmembrane (TM) helical segment from virus protein $\mathrm{U}$ $(\mathrm{Vpu})$. The models are used to study both orientation and energetics of this $\alpha$-helical peptide as a function of hydrophobic mismatch. We observe that increasing the degree of positive hydrophobic mismatch increased the tilt angle of Vpu. These findings agree well with experimental data and as such validate the solvation models used in this study.
\end{abstract}

Keywords Tilt angle $\cdot$ Implicit solvent model $\cdot$ Umbrella sampling $\cdot$ Molecular simulations $\cdot$ Hydrophobic interaction

When carrying out molecular dynamics simulations of biological systems with classical force fields (Karplus and McCammon 2002), the most accurate representation involves modeling both the macromolecules and the solvent explicitly at atomic-level detail. However, because the solvent molecules dominate in number, the majority of the calculation time is spent on solvent-solvent interactions.

\footnotetext{
A. Frank · I. Andricioaei $(\square)$

Department of Chemistry, University of California, Irvine, CA 92697, USA

e-mail: andricio@uci.edu
}

This therefore limits the feasible amount of conformational samples of the macromolecular solute one is interested in. The problem is particularly exacerbated when the macromolecule undergoes large conformational changes that require significant solvent equilibration time. To this end, much effort has been expended to develop implicit solvent models that would represent the important physical information on the solute-solvent interaction as some effective energy function obtained by formally integrating, under various approximating assumptions, over all the solute degrees of freedom (Roux and Simonson 1999; Feig and Brooks 2004; Brannigan et al. 2006). The fact that the solute degrees of freedom have been integrated out enables for an adiabatic (i.e., instantaneous) response of the solvent to any new conformation of the solute. This instantaneous equilibration is especially important when the macromolecule is solvated a lipid membrane environment, whose slow lateral and transversal reorganization requires relaxation on timescales that are very long and would require excessive computing in an explicit lipid representation (Pastor 1994; Forrest and Sansom 2000).

We focus on two implicit solvent models for lipid bilayer systems. The first model is implicit membrane model 1 (IMM1) (Lazaridis 2003), an extension for proteins in lipid bilayers surrounded by an aqueous environment of a previous effective energy function 1 (Lazaridis and Karplus 1999) developed to model proteins in aqueous solvents. IMM1 has been found to give good results with minimal CPU time and as such is quite promising. Specifically, IMM1 has been applied to the study of structure and energetics of TM proteins (Mottamal et al. 2006), insertion of helices into membranes (Lazaridis 2003), and helix orientation in lipid bilayers (Lazaridis 2003). The second model is the generalized Born with a simple switching model (GBSW) (Im et al. 2003). It builds on previous versions of generalized 
Born models as approximate solutions to the PoissonBoltzmann equation. Thus far, GBSW has been applied to the study of helix-helix interaction (Im et al. 2003), helix orientation in membranes, the role of the membrane in dictating conformational dynamics of TM proteins (Im et al. 2003), and interfacial folding of membrane proteins (Im and Brooks 2005). When compared to available experiment data, both IMM1 and GBSW agree reasonably well (see references for details).

Another challenging aspect in simulating biological systems is the determination of free energies changes for solvated systems (Levy and Gallicchio 1998). The majority of methods developed for this purpose are based on thermodynamic perturbation (Zwanzig 1954), thermodynamic integration (Kirkwood 1935), probability distribution methods (Bennett 1976; Torrie and Valleau 1977), or, more recently, the Jarzynski identity (Jarzynski 1997). A particularly useful approach in the category of the probability distribution methods is umbrella sampling (Bartels and Karplus 1997), which allows one to determine the free energies as a function of a predefined reaction coordinate. This is achieved by adding a potential to the Hamiltonian of the system that allows for the uniform sampling of conformational space as parametrized by the reaction coordinate. This methods has been successfully used in many cases, but the process can be time consuming. In cases when the reaction coordinate involves significant conformational displacement in a lipid environment, the ability to generate a uniform sampling during umbrella sampling along that coordinate relies heavily on the ability of the solvent to have sufficient time to respond to the extensive protein change. Such a solvent reorganization is computationally quite demanding, and of particular importance in instances involving the orientation of protein helices inside lipid bilayers. This problem of course does not exist in the case of implicit solvent/lipid models, for which - as a result of the integration over their degrees of freedom-the "solvent" and the "lipid" relax instantaneously in response to any change in orientation or structure.

Hydrophobic mismatch, defined as the difference between the hydrophobic length of TM segments of a protein and the hydrophobic width of the surrounding lipid bilayer, is the driving force behind TM segment reorientation in situations when the hydrophobic stretch of a membrane pass protein is not equal to the thickness of the hydrophobic core of the lipid bilayer (see Killian 1998 for a detailed review). Hydrophobic mismatch can be of two types: positive mismatch, when the hydrophobic stretch of the peptide is longer than the thickness of the hydrophobic core of the membrane, or negative, the case when the thickness of the hydrophobic core exceeds the peptide length. Regardless of its type, this mismatch yields an energetically unfavorable condition: in the case of positive mismatch, some of hydrophobic side chains can get exposed to solvent, and in the case of negative mismatch, some of the hydrophilic sides can find themselves buried within the hydrophobic core of the membrane. Stand-alone TM peptides or TM segments in membrane protein complexes rearrange such as to minimize the hydrophobic mismatch and to achieve hydrophobic matching (White and von Heijne 2005), although in particular instances, interfacial anchor properties of tryptophan residues in transmembrane (TM) peptides can dominate over hydrophobic matching effects in peptide-lipid interactions (de Planque et al. 2003).

In instances of positive mismatch, which is the case of this study, the TM helices rearrange by tilting relative to the membrane normal. Positive hydrophobic mismatching was of interest to us because of the significant amount of experiment data generated about this process. As such, it provided the opportunity to test the applicability of implicit solvent modeling by making a comparison to available experimental data.

The TM helix is the fundamental structural unit of helix bundle membrane proteins. A proper computational description of the free energy of association at the atomic detail is an important ingredient in understanding the details of the amino acid code used by the translocon to select polypeptide segments for insertion as TM helices in helix bundle membrane proteins (White and von Heijne 2005). A good model system for the purpose of our computational study is $\mathrm{Vpu}$, an accessory protein of HIV-1 with wellcharacterized biological activities; as such, it serves as a suitable subject for the development of methods for structure determination of membrane proteins (Park et al. 2003). Park and Opella (2005) reported liquid and solid-state nuclear magnetic resonance data to derive structural information on the orientation of $\mathrm{Vpu}$. They found that the tilt of a helix in a membrane (in their case the TM protein $\mathrm{Vpu}$ ) is dependent on positive hydrophobic mismatch. Specifically, they reported that cosine of the tilt angle, $\theta$, increases linearly with the degree of positive hydrophobic mismatch.

In this project, we tested the ability of IMM1 and GBSW to recreate the experimentally observing tilting of the TM helix Vpu in response to positive hydrophobic mismatch. Additionally, umbrella sampling (Bartels and Karplus 1997) was used to obtain free-energy profiles of the tilting as a function of membrane thickness. Assuming that the tilt angle a helix adopts in a membrane represents its energetically favorable orientation, by using these freeenergy profiles, we can predict the tilt angle. We can then compare these to the experimental values, and as such evaluate the performance of IMM1 and GBSW solvent models in concert with umbrella sampling. These are critical tests because the tilting requires that the protein 
systems undergo sweeping motions involving large excursions inside the lipid.

We present the results of this simple study, which show that IMM1 and GBSW are suitable membrane models for carrying out molecular dynamics simulation of simple protein-lipid systems by the fact that umbrella samplingbased free-energy profiles generate hydrophobic mismatch patterns in accord with experimental data.

\section{Methods}

The membrane is modeled as a lipid slab between two water regions using two implicit solvent models. The first one, IMM1, uses an empirical energy function, parameterized with experimental data. IMM1 primarily accounts for solvent exclusion effects and assumes that total solvation energy is the sum of group contributions. It partially accounts for electrostatic interaction by using a distance dependent dielectric function allowing for screening of electrical charge. The second model, GBSW, decomposes the solvation free energy into nonpolar and electrostatic components. The nonpolar component is approximated as being proportional to the accessible surface area of the protein. The electrostatic component is determined by an analytical approximation of the Poisson-Boltzmann method by a generalized Born equation for the calculation of the solvation energy for an isolated charge in some dielectric medium.

In our simulations, initial coordinates were obtained from the X-ray structure of TM segment of Vpu (PDB ID: 1pje). For both IMM1 and GBSW, four independent simulations were carried out with a membrane thickness $D$ equal to $15,19,23$, and $27 \AA$, respectively. For each simulation, the following protocol was used. The initial structure was subjected to 100 steps of steepest-descent minimization followed by 500 steps of adopted-basis Newton-Raphson minimization. The system was centered at the midpoint of the membrane as the origin and the helical axis of $\mathrm{Vpu}$ aligned to the $z$-axis (the normal to the membrane plane). The system was heated from 0 to 298.15 K. Subsequently, a 60-ns trajectory was generated using Langevin dynamics. Frictional constant of $91 \mathrm{ps}^{-1}$ was used. The SHAKE (Ryckaert et al. 1977) algorithm was used to constrain bonds involving hydrogen, allowing for a 0.002 -ps integration time step.

Free-energy profiles for the tilt angle $\theta$ relative to the $z$-axis were determined by means of umbrella sampling and the weighted histogram analysis method (WHAM). This method has been extensively used to determine free-energy profiles, and as such, the details related to theoretical background and implementation are not included here (see Kumar et al. 1992; Roux 1995). Briefly, umbrella sampling involves applying a bias to restrict sampling along some reaction coordinate. To obtain potential of mean force (PMF) profiles, the reaction coordinate is divided into a number of sampling windows, and in each window, simulations are biased to sample conformations around some value of the reaction coordinate. Statistics are collected in each window, and then WHAM is used to unbias and combine statistics from all windows to generate the desired PMF profiles along the entire range of the reaction coordinate. In this study, the biasing potential was of the following form:

$V(\theta)=\frac{k}{2}\left(\theta-\theta_{0}\right)^{2}$,

where $k$ is the force constant, $\theta$ is the tilt angle (defined as the angle subtended by the vector along the helical and the vector normal to the membrane; Fig. 1), and $\theta_{0}$ is the value of the tilt angle around which sampling is desired. Statistics were collected in 36 sampling windows in the range $\theta_{0}=[0,90]$, with a bin size of $2.5^{\circ}$. In a typical window, a force constant of $250 \mathrm{kcal} / \mathrm{mol} / \mathrm{deg}^{2}$ was used. Simulation in each window was $4 \mathrm{~ns}$ in length. The first $1 \mathrm{~ns}$ was discarded, and the other 3 ns was used to collect statistics.

\section{Results and Discussion}

The variation of the helix tilt as a function of time and membrane thickness, $D$, is summarized in Fig. 2. For simulations using IMM1 (Fig. 2a) and GBSW (Fig. 2b), the helix quickly tilts in response to positive hydrophobic mismatch, and it thereafter fluctuates about their mean. In the case of IMM1, as the membrane thickness is decreased from 27 to $15 \AA$, the tilt angle, measured as the time average of the length of the trajectory, increased from $9^{\circ}$ to $45^{\circ}$ (Fig. 2a). Similarly, in the case of GBSW, as the membrane thickness is decreased from 27 to $15 \AA$, the tilt angle increased from $18^{\circ}$ to $56^{\circ}$ (Fig. 2b). For comparison,

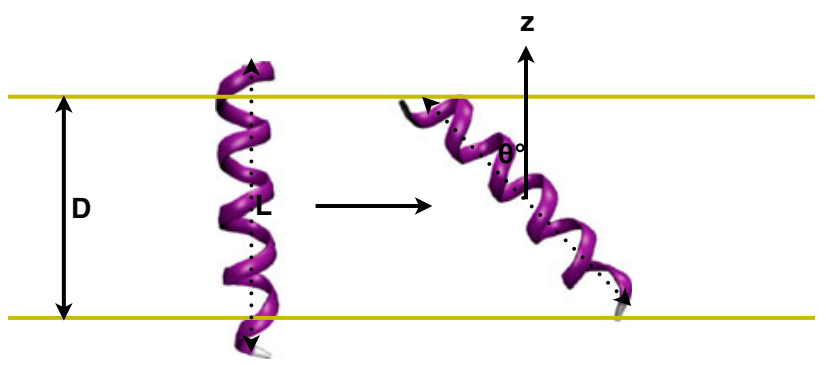

Fig. 1 Protein reorientation in response to hydrophobic mismatch. Schematic representation of the tilting of a TM protein under conditions of hydrophobic mismatch when membrane thickness $(D)$ is smaller than the length of the helix $(L)$. In response to mismatch, the helix tilts relative to the normal of the membrane (here the $z$ direction) such that hydrophobic side chains are buried inside the membrane 

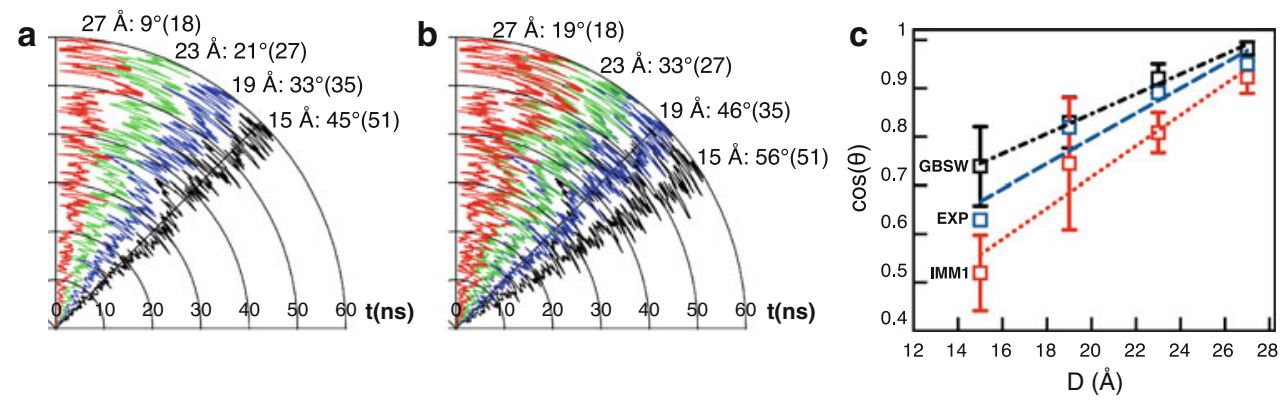

Fig. 2 Tilt angle time series. Tilt angles are shown as a function of time for Vpu simulated in the IMM1 (a) and GBSW (b) implicit membrane models. Results are shown for simulations where $D$ (membrane thickness) is 15 (black), 19 (blue), 23 (green), and $27 \AA$ A (red).

the corresponding experimentally determined tilt angles are shown in parentheses. These values show very good agreement with mean tilt angles for both IMM1 and GBSW. When $\cos \theta$ is plotted against $D$, a roughly linear relationship is revealed, in agreement with Park and Opella (2005) (Fig. 2c). These results corroborate those of other molecular dynamics simulation studies on $\alpha$-helical TM proteins. Specifically, using an explicit lipid representation for the membrane, Goodyear et al. (2005) observed that for a synthetic peptide initially oriented along the normal of membrane the helix tilts in response to positive hydrophobic mismatch, with an average tilt angle comparable to the experimentally determined value.

The next phase of the study involved the determination of free-energy profiles as a function of the tilt of the $\alpha$-helical peptide. By means of umbrella sampling, the free energy as a function of tilt angle was determined for angles ranging from $0^{\circ}$ to $90^{\circ}$ for membrane thicknesses of 15,19 , 23, and $27 \AA$, respectively. As mentioned earlier, if we assume that the optimal tilt angles a helix adopts in a membrane represent its energetically favorable orientations according to the free energy computed with the implicit solvent model, then by using these free-energy profiles, we can predict tilt angles. In the case of IMM1, for $D=15$,
Shown on each plot are the average angles. For comparison, experimental tilt angles are shown in parentheses. Shown in (c) is a plot of $\cos (\theta)$ vs. $D$ determined using results from IMM1 simulation (red), GBSW (black) simulation, and experiment (blue) (Color figure online)

19,23 , and $27 \AA$, the absolute minimum was located at $41^{\circ}, 31^{\circ}, 17^{\circ}$, and $11^{\circ}$, respectively (Fig. 3a). In the case GBSW, for $D=15,19,23$, and $27 \AA$, the absolute minimum was located at $51^{\circ}, 41^{\circ}, 29^{\circ}$, and $14^{\circ}$, respectively (Fig. 3b).

The fact that the results obtained from the IMM1 and GBSW models so closely resemble the experiment is significant because it implies that the implicit models used for this system contained all the necessary physics as it relates to electrostatic and hydrophobic interaction within the membrane region. Although more detailed interactions such as anchoring interactions between peptides and lipids (e.g., tryptophan residues interacting with the polar head group region; de Planque et al. 2003) can compete with the fundamental free-energy driving force captured in IMM1 and GBSW, we believe that our study shows that IMM1 and GBSW, at least for systems as simple as the one used in this project, certainly embody the necessary functional form and parameters to describe the tilt process as a function of positive hydrophobic mismatch. IMM1 and GBSW thus prove promising for membrane proteins, given the savings in computer time, while at the same time accurately recreating the solvent effects of the cell membrane and the surrounding aqueous regions.
Fig. 3 Potential of mean force profiles. Free-energy profiles are shown as a function of tilt angle $(\theta)$ calculated using the IMM1 (a) and GBSW (b) implicit membrane models. Shown are profiles calculated where $D$ (membrane thickness) is 15 (black), 19 (blue), 23 (green), and $27 \AA$ (red) (Color figure online)
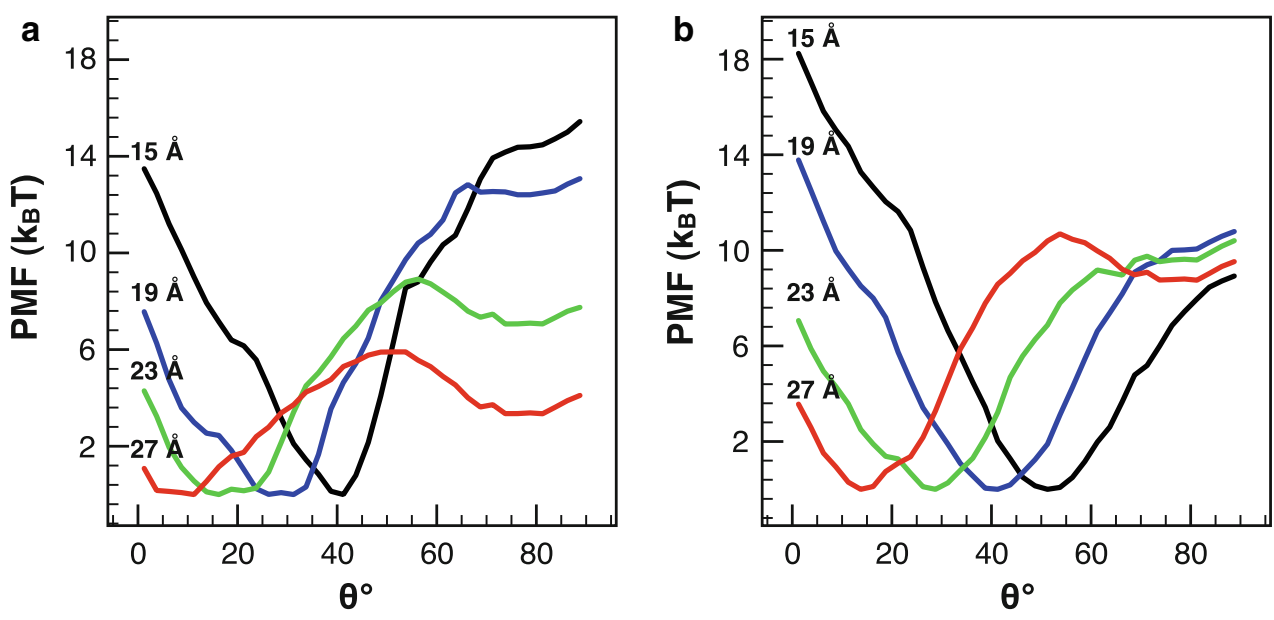
A comparison between IMM1 and GBSW reveals that they yield qualitatively similar results. They were both able to recreate the tilting of the $\alpha$-helical TM peptide Vpu as function of hydrophobic mismatch that was observed experimentally, and they both exhibit a linear relationship between $\cos \theta$ and $D$, in agreement with Park and Opella (2005). Quantitatively, however, IMM1 and GBSW give slightly different tilt angles for a given membrane thickness. In general, IMM1 gave values lower than the experimental observed values, while GBSW gave higher values (Fig 2). This is also reflected in the free-energy profile, where qualitatively the models mirrored each other, but quantitatively there were slight difference (GBSW predicted the minima to exist at higher $\theta$ for any given $D$; Fig. 3). The PMFs for IMM1 and GBSW also differ as the tilt angle approaches $80^{\circ}$. For IMM1, the barrier to tilting beyond the minima is larger as the membrane thickness decreases. The trend is reversed slightly in the case of GBSW, but in this region, the difference amounts to a few $\mathrm{kcal} / \mathrm{mol}$.

In terms of the performance of the algorithms, simulations using IMM1 were on average four times as fast as GBSW. This can be attributed to the fact that GBSW involves the time-consuming calculation of the accessible surface area, which is used to approximate the nonpolar contribution to the solvation energy.

An intriguing knowledge-based potential by Ulmschneider et al. (2005) was derived and recently applied to the Vpu helix (Ulmschneider et al. 2006). The basic hypothesis of that study was that each residue on its own would prefer a certain depth inside the membrane bilayer. Potentials of mean force along the membrane normal were derived for each amino acid by fitting Gaussian functions to residue position distributions from known TM structures. The individual potentials agreed well with experimental and theoretical considerations. The resulting implicit membrane potential was tested on various membrane proteins as well as single trans-membrane helices. All membrane proteins were found to be at an energy minimum when correctly inserted into the membrane. The results of that study qualitatively match those obtained in our study.

\section{Conclusions}

In our computational test, the use of two widely used implicit membrane models, IMM1 and GBSW, was sufficient to reproduce the experimentally measured tilting (Park and Opella 2005) of an $\alpha$-helical peptide that occurs in response to positive hydrophobic mismatch. Umbrella sampling-based free-energy profile calculations to obtain the distribution of the peptide tilt angles relative to the membrane proved to be a reliable means of gaining information concerning the free energy of the system.

Acknowledgments ATF was supported by the National Science Foundation Graduate Fellowship program. IA acknowledges support from the National Science Foundation Career award (CHE-0918817).

Open Access This article is distributed under the terms of the Creative Commons Attribution Noncommercial License which permits any noncommercial use, distribution, and reproduction in any medium, provided the original author(s) and source are credited.

\section{Reference}

Bartels C, Karplus M (1997) Multidimensional adaptive umbrella sampling: applications to main chain and side chain peptide conformations. J Comput Chem 18:1450-1462

Bennett CH (1976) Efficient estimation of free-energy differences from Monte-Carlo data. J Comput Phys 22:245-268

Brannigan G, Lin LCL, Brown FLH (2006) Implicit solvent simulation models for biomembranes. Eur Biophys J Biophys Lett 35:104-124

de Planque MRR, Bonev BB, Demmers JAA, Greathouse DV, Koeppe RE II, Separovic F, Watts A, Killian JA (2003) Interfacial anchor properties of tryptophan residues in transmembrane peptides can dominate over hydrophobic matching effects in peptide-lipid interactions. Biochemistry 42:5341-5348

Feig M, Brooks CL (2004) Recent advances in the development and application of implicit solvent models in biomolecule simulations. Curr Opin Struct Biol 14:217-224

Forrest LR, Sansom MSP (2000) Membrane simulations: bigger and better. Curr Opin Struct Biol 10:174-181

Goodyear DJ, Sharpe S, Grant CWM, Morrow MR (2005) Molecular dynamics simulation of transmembrane polypeptide orientational fluctuations. Biophys J 88:105-117

Im W, Brooks CL (2005) Interfacial folding and membrane insertion of designed peptides studied by molecular dynamics simulations. Proc Natl Acad Sci U S A 102:6771-6776

Im W, Feig M, Brooks CL (2003) An implicit membrane generalized born theory for the study of structure, stability, and interactions of membrane proteins. Biophys J 85:2900-2918

Jarzynski C (1997) Nonequilibrium equality for free energy differences. Phys Rev Lett 78:2690-2693

Karplus M, McCammon JA (2002) Molecular dynamics simulations of biomolecules. Nat Struct Biol 9:646-652

Killian JA (1998) Hydrophobic mismatch between proteins and lipids in membranes. Biochim Biophys Acta 1376:401-416

Kirkwood JG (1935) Statistical mechanics of fluid mixtures. J Chem Phys 3:300

Kumar S, Bouzida D, Swendsen RH, Kollman PA, Rosenberg JM (1992) The weighted histogram analysis method for free-energy calculations on biomolecules. I. The method. J Comput Chem 13:1011-1021

Lazaridis T (2003) Effective energy function for proteins in lipid membranes. Proteins 52:176-192

Lazaridis T, Karplus M (1999) Effective energy function for proteins in solution. Proteins 35:133-152

Levy RM, Gallicchio E (1998) Computer simulations with explicit solvent: recent progress in the thermodynamic decomposition of free energies and in modeling electrostatic effects. Annu Rev Phys Chem 49:531-567

Mottamal M, Zhang J, Lazaridis T (2006) Energetics of the native and non-native states of the glycophorin transmembrane helix dimer. Proteins 62:996-1009 
Park SH, Opella SJ (2005) Tilt angle of a trans-membrane helix is determined by hydrophobic mismatch. J Mol Biol 350:310-318

Park SH, Mrse AA, Nevzorov AA, Mesleh MF, Oblatt-Montal M, Montal M, Opella SJ (2003) Three-dimensional structure of the channel-forming trans-membrane domain of virus protein " $u$ " (Vpu) from HIV-1. J Mol Biol 333:409-424

Pastor RW (1994) Molecular dynamics and Monte Carlo simulations of lipid bilayers. Curr Opin Struct Biol 4:486-492

Roux B (1995) Calculation of the potential of mean force using computer simulations. Comput Phys Commun 91:275-282

Roux B, Simonson T (1999) Implicit solvent models. Biophys Chem 78:1-20

Ryckaert JP, Ciccotti G, Berendsen HJC (1977) Numerical integration of the Cartesian equations of motion of a system with constraints: molecular dynamics of n-alkanes. J Comput Phys 23:327-341
Torrie GM, Valleau JP (1977) Non-physical sampling distributions in Monte-Carlo free-energy estimation-umbrella sampling. J Comput Phys 23:187-199

Ulmschneider MB, Sansom MSP, Di Nola A (2005) Properties of integral membrane protein structures: derivation of an implicit membrane potential. Proteins 59:252-265

Ulmschneider MB, Sansom MSP, Di Nola A (2006) Evaluating tilt angles of membrane-associated helices: comparison of computational and NMR techniques. Biophys J 90:1650-1660

White SH, von Heijne G (2005) Transmembrane helices before, during, and after insertion. Curr Opin Struct Biol 15:378-386

Zwanzig RW (1954) High-temperature equation of state by a perturbation method. I. Nonpolar gases. J Chem Phys 22:1420 